\title{
Optimisation procedure for additive manufacturing processes based on mask image projection to improve $Z$ accuracy and resolution
}

\author{
J. Bonada (1)(2) *, A. Muguruza(1), X. Fernández-Francos(3), X. Ramis(3)
}

(1) Centre CIM, Universitat Politècnica de Catalunya, Barcelona, Spain

(2) Strength of Materials and Structural Engineering Department, ETSEIB, Universitat Politècnica de Catalunya, Barcelona, Spain

(3) Thermodynamics Laboratory, ETSEIB, Universitat Politècnica de Catalunya, Barcelona, Spain.

\begin{abstract}
Mask image projection is an additive manufacturing (AM) technique used in photocurable materials which allows the simultaneous energy delivery in a whole area instead of a single spot. A common problem for this AM process is the uncontrolled penetration of light energy, which could cause a solidification of non-desired layers. In this paper, an optimization procedure is developed and presented in order to increase $Z$ accuracy and resolution of printed parts through the control of the total accumulated dose and the photocuring conversion ratio of each spatial location of the manufacturing volume. Consequently, the uncontrolled monomer-to-polymer conversion of down-facing surfaces could be reduced. A finite element strategy is used in the optimisation procedure to obtain a full discretization of the whole manufacturing domain. Furthermore, experimental tests have been done to compare experimental results and numerical estimations. The results show that the use of the optimisation procedure increases the accuracy and resolution of printed parts along the manufacturing direction.
\end{abstract}

Keywords: Additive manufacturing; Mask image projection; manufacturing optimisation

*Corresponding author. Tel.: +34 934017171
E-mail addresses: jbonada@fundaciocim.org (J. Bonada) 


\section{Introduction}

Additive manufacturing (AM) processes are used to build-up solid parts by a sequential addition of material layer by layer. During the last decades, several AM technologies have been developed such as selective laser sintering (SLS), selective laser melting (SLM), stereolithography (SLA), fused deposition modelling (FDM), etc. AM processes [1, 2] allow manufacturing solid parts directly from the digital model (STL file [3]) without using specific fixtures or tools; therefore they have been traditionally used for rapid prototyping.

Frontal photopolymerization (FPP) $[4,5]$ is an unidirectional process in which a photocurable material is solidified along the manufacturing direction as a consequence of a light exposure dose. FPP is used in several AM techniques, such as SLA or mask image projection based stereolithography (MIP-SL). Previous research has been done in order to develop a frontal photopolymerisation analytical model. In [6], a FPP model to determine the photocuring conversion ratio of photocurable materials in function of the received exposure dose and material parameters is presented. Furthermore, photocuring conversion has a significant influence in material mechanical properties such as density, shrinkage, Young's modulus, ultimate stress, etc. [7, 8]. Therefore, a non-uniform conversion ratio could cause non-desired consequences in printed parts.

Mask image projection based stereolithography (MIP-SL) uses a Digital Light Processing (DLP) system, based on a Digital Micromirror Device (DMD), to manage light exposure on a photocurable material [9, 10]. This technology allows a simultaneous energy delivery in the whole manufacturing area. Thus, the manufacturing speed could be higher than traditional SLA which delivers the energy through a single spot using a UV-laser and a scanning process. However, the resolution and accuracy of MIP-SL is limited by the number of mirrors in the DMD. In [11], Zhou and Chen presented a novel AM process based on mask-video-projection, which extends the optimised pixel blending principle [12] to increase the inplane resolution by means of several multiple mask images for each layer. In order to determine the mask image planning process an optimisation procedure was required and developed [11, 12]. They use a heuristic optimisation method to obtain a sub-optimal solution. The proposed method was validated by different experimental tests.

Several other alternatives have been presented in order to increase accuracy. In [13], a process planning method to properly define part orientation, slicing scheme and printing parameters for stereolithography was presented in order to assist the SLA user. Zha and Anand [14] developed a Surface based Modification Algorithm (SMA) which adapts and locally increase the facet density of STL model in order to decrease geometrical errors. Thus, each of the local original STL facets modified by SMA algorithm is replaced by a number of new facets. Moreover, obtaining smooth surfaces has also been a research focus. In [15], a methodology to smooth STL files for rapid prototyping medical models was implemented. A Fast Fourier Transform (FFT) interpolation algorithm was introduced to improve the accuracy of the model. The results were validated in built-up parts using FDM technology. On the other hand, in [16] a mask image projection process has been developed for manufacturing smooth upfacing surfaces via a meniscus equilibrium method. Experimental tests have been used to validate the projection image planning in concave and convex geometries.

A common issue with AM technologies based on FPP such as SLA or MIP-SL is the uncontrolled penetration of light energy into the photocuring material $[17,18]$. Consequently, a non-desired material solidification is produced in down-facing surfaces. Traditionally, a modification of down-facing surfaces in STL file has been done to compensate this effect. However, Choi et al. [19] presented a research in which different concentrations of light absorbers were used to reduce light penetration and to achieve a successful manufacture of complex 3D microstructures with down-facing surfaces. Furthermore, Limyae and Rosen [20] presented a method called Compensation Zone approach to reduce the printing errors in the manufacturing direction in mask projection stereolithography. This method entails subtracting a tailored volume from the CAD model to compensate the increase of $Z$ dimensions of down-facing surfaces. In [21], Sager and Rosen presented a process planning based on parameter estimation (PE) method to improve surface accuracy of parts obtained through a SL equipment. The scan velocity of SLA 
process was used as a main variable to minimize the deviation of the received exposure dose from the critical energy.

The optimisation methods to increase Z accuracy based on a STL modification could produce good results in several AM technologies. However, their efficiency could be reduced in AM photopolymerisation processes if the uncontrolled penetration of the exposure dose as well as the manufacturing process planning is not modelled and considered. In the present paper, a novel analytical optimisation method for MIP-SL based on a finite element discretization is presented and implemented in order to minimize the difference between the objective and the achieved photoconversion ratio of printed parts. Therefore, this novel methodology allows the minimization of printing errors in the manufacturing direction of down-facing surfaces taking into account the total accumulated dose received in any spatial location during the manufacturing process. Furthermore, this methodology also allows defining an objective photocuring conversion ratio for any part location. Consequently, it is possible to define higher conversion ratios for printed parts in order to reduce or avoid a UV post-curing process and to obtain a full photocuring conversion. Moreover, it could improve the conversion ratio uniformity along the manufacturing direction, which it would avoid the presence of different mechanical properties or shrinkage along $\mathbf{Z}$ direction. The analytical optimisation method is validated for photosensitive materials based on acrylate oligomers through several experimental tests in down-facing surfaces. The proposed optimisation method could be used and implemented for any photocurable material and MIP-SL equipment based on a Frontal Photopolymerization Process (FPP). The light source of MIP-SL equipment has to be able to project greyscale mask images.

\section{Photopolymerisation model}

\subsection{Analytical Model}

The photo-invariant FPP model presented and developed in [6-8] describes the spatio-temporal photoconversion $(\chi)$ evolution of a photocurable resin. The extent of photopolymerization along the manufacturing direction $(\Phi(z, d))$ is a dimensionless parameter $\left(\Phi=\chi / \chi_{\max }\right)$ used to describe the monomer-to-polymer conversion (Eq.1):

$\phi(z, d)=1-\exp (-K d \exp (-\mu z))$

where $z$ is the manufacturing direction (normal to the irradiated surface), $K$ is the material effective reaction conversion rate, $d$ is the exposure dose and $\mu$ is the attenuation coefficient. The FPP model is assumed to be photo-invariant; thus the attenuation coefficient is independent of the conversion ratio. Furthermore, the gel point of a photosensitive material corresponds to a threshold conversion ratio value $\left(\Phi_{c}\right)$. If the conversion ratio achieved is higher than $\Phi_{c}$ a network is formed and a solidified material layer is obtained. For a single exposure, the solidified thickness $\left(Z_{f}\right)$ could be obtained through Eq. 2.

$Z_{f}=\frac{\ln \left(\frac{K \cdot d}{\ln \left(\frac{1}{1-\phi_{C}}\right)}\right)}{\mu}$

Other research has been done to take into account mass and thermal effects [22] as well as oxygen inhibitory effect [23] in analytical models. Thermal effects could only have a significant influence on FPP kinetics if there is a temperature increment above $50 \circ \mathrm{C}$ during the photopolymerisation reaction. The oxygen inhibitory effect is only important in a few microns of depth, thus it is mainly modelled in microfabrication. Therefore, these two effects have not been considered in this paper. 


\subsection{Model calibration}

The main parameters of FPP model were obtained by means of experimental tests. The same light source of MIP-SL equipment was used to irradiate the photocurable resin used in this paper with different exposure doses to obtain several samples with different thicknesses. Therefore, the solidified thickness dependence on logarithmic light exposure dose for the samples is presented in Fig. 1 (a) and Table 1. Consequently, the attenuation factor is calculated as the inverse of the experimental slope. Three different samples for each case has been obtained. The samples thicknesses were measured (Fig. 1(b)) via $2 \mathrm{~mm}$ displacement transducer ( $0.2 \%$ of linearity deviation). The results show a small experimental deviation for each exposure dose.

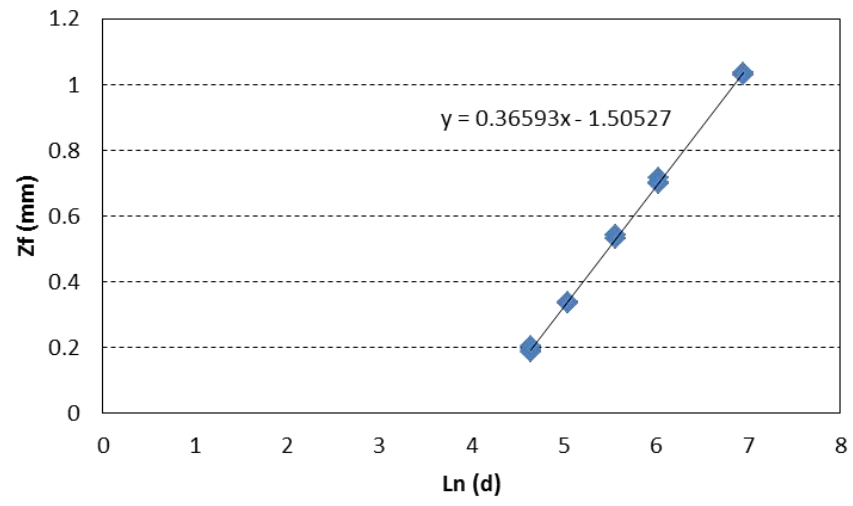

(a)

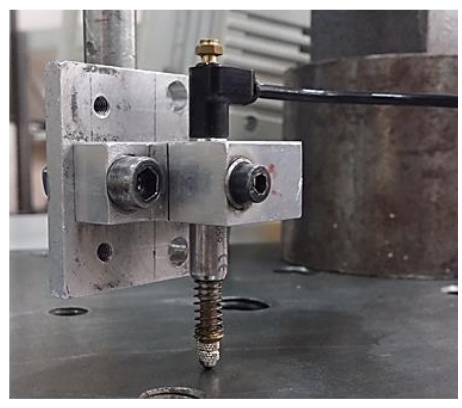

(b)

Figure 1 - Samples thicknesses present a proportional relationship $(1 / \mu)$ with logarithmic dose (a). Experimental equipment used to measure the thickness of the samples (b).

\begin{tabular}{cccc}
\hline Exposure dose $\left(\mathrm{mj} / \mathrm{cm}^{2}\right)$ & Solidified thickness $\left(Z_{f}\right)(\mathrm{mm})$ & Mean & Deviation \\
\hline 104 & 0.192 & & \\
104 & 0.185 & 0.193 & 0.009 \\
104 & 0.203 & & \\
\hline 156 & 0.335 & 0.003 \\
156 & 0.340 & & \\
156 & 0.335 & 0.007 \\
\hline 260 & 0.530 & 0.537 & \\
260 & 0.540 & & \\
260 & 0.542 & & \\
\hline 416 & 0.699 & 0.009 \\
416 & 0.715 & & \\
416 & 0.700 & & \\
\hline 1040 & 1.036 & & \\
1040 & 1.030 & & \\
1040 & 1.033 & & \\
\hline
\end{tabular}

Table 1 - Experimental values of the calibration samples.

The threshold conversion ratio value or gel point $\left(\Phi_{c}=0.276\right)$ was measured by means of FTIR spectrometry bands of acrylate groups (Fig. 2(a)) for all printed samples. The normalized peak area of the absorption band of $810 \mathrm{~cm}^{-1}$ was used to determine the conversion ratio. The $1730 \mathrm{~cm}^{-1}$ band was used as a reference in Eq. 3. 
$\chi=1-\frac{A_{d}^{810} / A_{d}^{1730}}{A_{0}^{810} / A_{0}^{1730}}$

$A_{d}$ and $A_{0}$ indicates the absorption band peak area for the resin after and before received a controlled exposure dose, respectively. Fig. 2(b) shows the FTIR spectrometry at $810 \mathrm{~cm}^{-1}$ band for non-converted resin and for a printed sample at $Z_{f}$ location, where the threshold conversion ratio is determined.

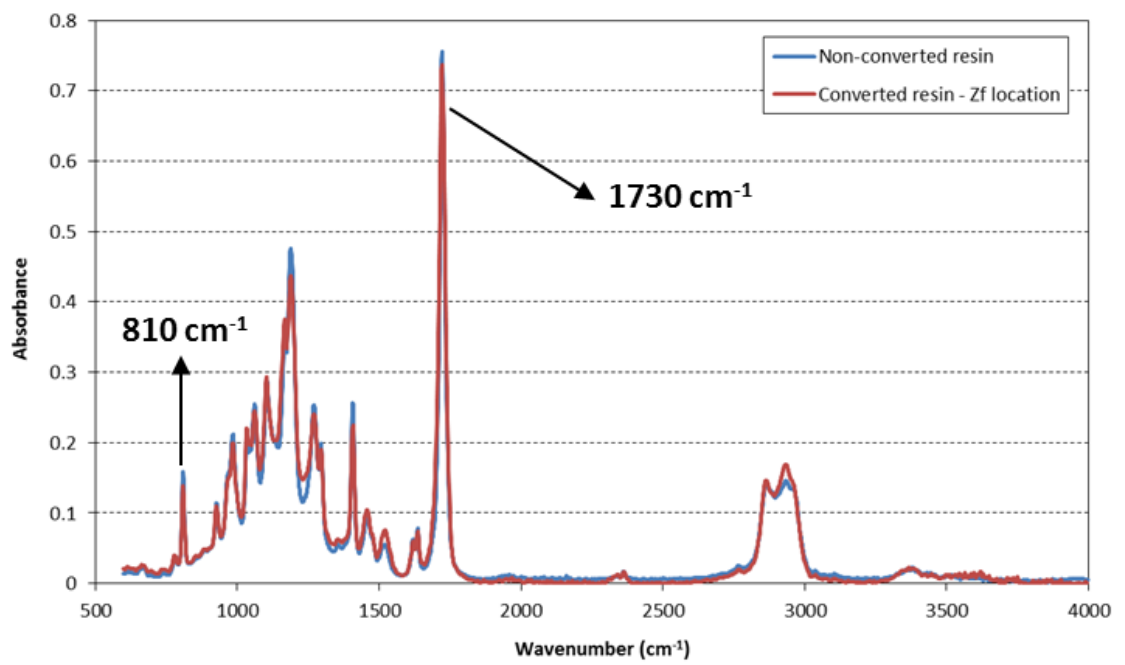

(a)

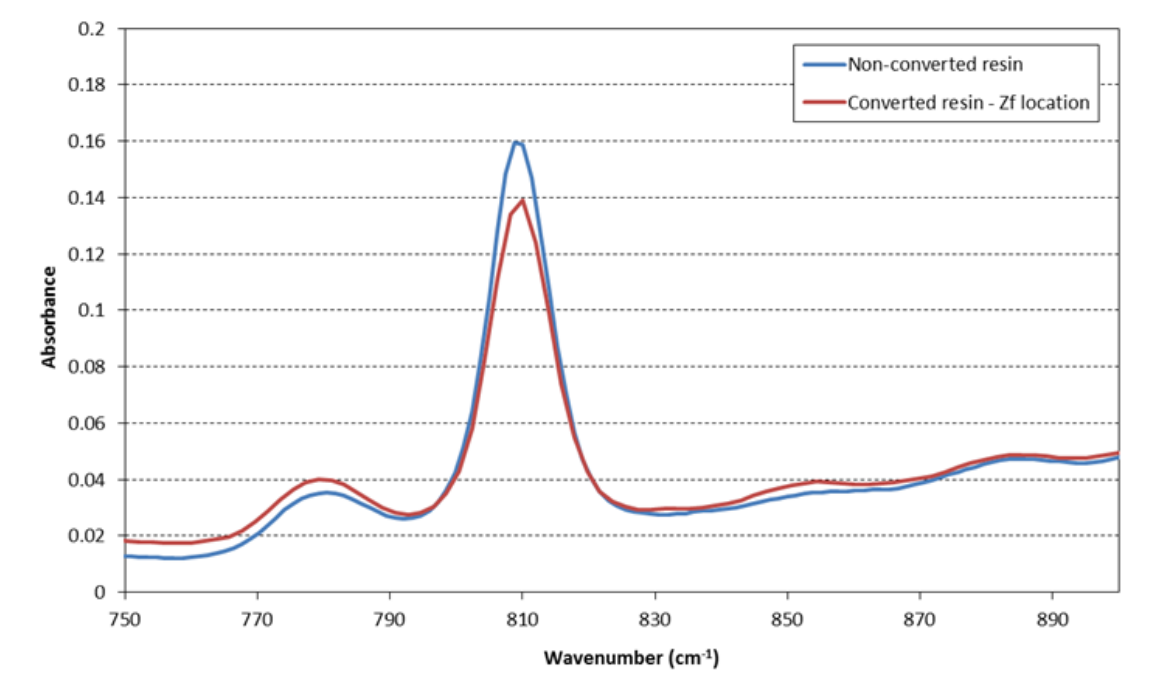

(b)

Figure 2 - FTIR spectrometry results (a). Detail of $810 \mathrm{~cm}^{-1}$ absorption band, where the threshold conversion ratio is calculated (b).

The authors attempted to relate these results with experimental gel-point measurements carried out following the procedures described in [24]. Different thermal initiators were added to the formulation in different proportions and the mixtures were heated in a thermomechanical analyser (TMA, Mettler TMA840) until gelation was observed. Direct comparison of the gel point with samples cured in a differential scanning calorimeter (DSC, Mettler DSC821e) at the same heating rate did not provide meaningful results because of the difference in sample preparation and the different environments in the TMA and DSC ovens. However, analysis of the SDTA signal (Single Differential Thermal Analysis) in the TMA revealed that gelation took place at the onset of the curing process. Although we could not determine an exact value of the conversion at the gel point, this suggested that gelation took place at the very beginning of the curing process, as expected for ring-free, chain-wise radical polymerisation of 
multifunctional monomers [25-26]. Fig. 2 shows that the degree of conversion measured by FTIR/ATR in the samples is clearly higher and measurable $(27.6 \%)$. The reasons behind this discrepancy could be manifold: (i) the conversion of deeper layers with higher conversion were measured due to the insufficient mechanical strength of the lightly crosslinked gel and the load pressure applied to carry out the measurement in the FTIR/ATR equipment; (ii) the fact that in the ATR measurement the evanescent wave penetrates the sample a number of microns and (iii) extremely lightly gelled layers are eventually washed away during processing due to their very poor mechanical strength and poor adhesion to the above layers (or are affected by microgelation phenomena [26]) so that, from a practical point of view, only layers with a sufficient degree of conversion and mechanical strength can become effectively incorporated into the processed parts. Consequently, a threshold conversion clearly above the gel point could be reasonably expected; thus, the conversion measured by FTIR/ATR can be confidently used as a valid parameter in the analytical model.

The parameters of FPP model obtained through the calibration procedure could be used for every new print with different part geometries or manufacturing settings. However, each photocurable material could present different FPP parameters such as attenuation factor, material effective reaction conversion rate or threshold conversion ratio. The use of a different light source could also modify some FPP parameters according to its wavelength and the photocurable material absorption spectrum. Therefore, the calibration procedure should be repeated in order to find the new FPP parameters if a different photocurable material or a different light source is used.

\subsection{Non-optimised additive manufacturing process}

When a non-optimised mask image projection based on stereolithography (MIP-SL) process is used all mask images are emitted with the same exposure dose $\left(d_{0}\right)$. As a result, the first layers will receive more accumulated doses than the last ones. This effect is shown in Fig. 3. Once the first layer is done, the second layer receives the same exposure dose $\left(d_{0}\right)$; however the first layer also receives an additional non-desire dose $\left(\Delta d_{2}\right)$, which can be calculated through Eq. 4.

$\Delta d_{j}=d_{o} \exp \left(-\mu \Delta z_{j}\right)$

A stepped conversion ratio profile is obtained and an uncontrolled monomer-to-polymer conversion could be produced in certain material zones if their conversion ratio exceeds the threshold conversion ratio. Consequently, $\mathrm{Z}$ accuracy or system resolution could be reduced by means of non-desired conversions. A traditional strategy to avoid this effect is based on the STL data modification of the down-facing areas to compensate the solidification of non-desired layers. However, the overcuring depends on the total accumulated doses of each spatial location; therefore it could be unequal in the entire manufacturing domain. Consequently, an optimisation procedure, which determines a specific exposure dose for each pixel of each layer to control the photoconversion ratio and to enhance $Z$ accuracy and resolution is presented in the following section. 


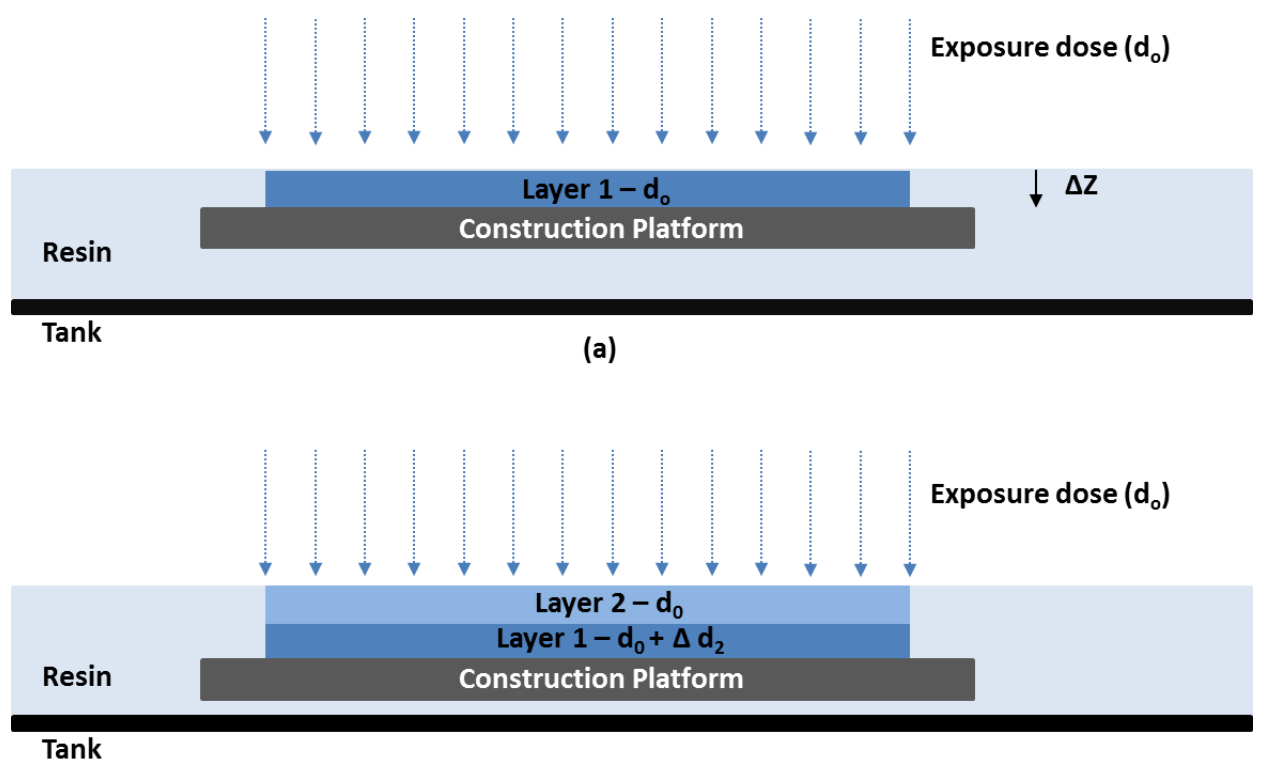

(b)

Figure 3 -During the first manufacturing step layer 1 receives an exposure dose (a). In following steps it could receive additional doses due to the lack of dose attenuation (b)

\section{Manufacturing process optimisation}

\subsection{Development of a discrete model}

The construction volume has been divided in several subdomains using a finite element discretization strategy (Fig. 4(a)) in order to optimise the manufacturing process along manufacturing direction. The following assumptions have been considered: (i) the photopolymerisation is only spread along positive $Z$ axis (Fig. 4(b)), (ii) the exposure dose is constant in a single pixel domain $\left(P_{i}\right)$ and (iii) the monomer-topolymer conversion process transmission along $X$ or $Y$ direction is neglected. Consequently, the extent of photopolymerization $(\Phi(z, t))$ for each node on any element for the pixel domain $\left(P_{i}\right)$ can be obtained through Eq. 5 and 6.
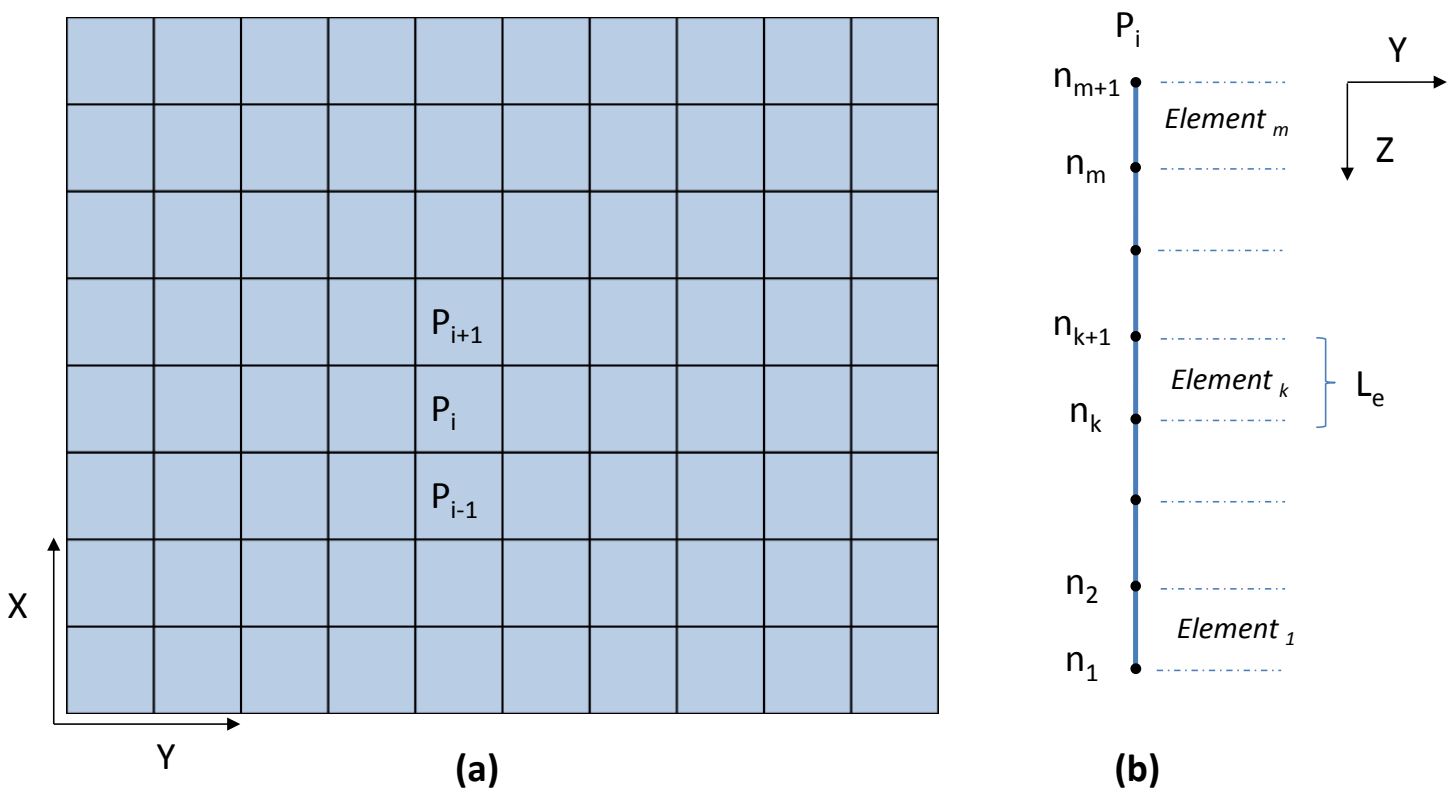

(b)

Figure 4. (a) Discretization in XY plane. Each domain corresponds to one pixel (Pi). (b) Discretization along Z axis for a single pixel domain $\left(P_{i}\right)$ using $m$ elements and $m+1$ nodes. 
A 2-node finite element has been formulated with one external load and one degree of freedom (d.o.f) for node $\left(d_{k, P_{i}}\right.$ and $\varphi_{k}$ respectively); where $d_{k, P_{i}}$ is the applied exposure dose on node $k$ for pixel domain $P_{i}, L_{e}$ the element length and $\varphi_{k}$ is a photopolymerisation process variable (Eq. 6), which is used to linearize FPP model.

Element $_{k} \rightarrow \varphi_{k+1}=-K \sum_{j=k+1}^{m+1}\left(d_{j, P_{i}} \cdot \exp \left(-\mu(j-(k+1)) L_{e}\right)\right)$

Element $_{k} \rightarrow \varphi_{k}=-K \sum_{j=k+1}^{m+1}\left(d_{j, P_{i}} \cdot \exp \left(-\mu(j-(k+1)) L_{e}\right)\right) \exp \left(-\mu \cdot L_{e}\right)$

$\varphi_{k}=\ln \left(1-\Phi_{k}\right)$

The phtopolymerisation process of the entire system can be presented in a matrix form (Eq. 7); where $[\varphi]_{P_{i}},[T]$ and $[d]_{P_{i}}$ corresponds to photopolymerisation variable (d.o.f), transmission matrix and nodal exposure dose for the pixel domain $\left(P_{i}\right)$; respectively.

$[\varphi]_{P_{i}}=[T] \cdot[d]_{P_{i}}$

$T_{p r}=-K \cdot \exp \left[-\mu(p-r) L_{e}\right] \rightarrow p \geq r$

$T_{p r}=0 \rightarrow p<r$

\subsection{Optimisation procedure}

An iterative algorithm based on gradient descent is used to optimise the exposure dose of each pixel of each layer to achieve an objective photocuring conversion ratio for each node and to reduce dimensional errors along the manufacturing direction. The objective function to be minimized can be seen in Eq. 8. The solution is obtained through the iterative method defined by Eq. 9. This procedure has to be repeated for each pixel domain $\left(\mathrm{P}_{\mathrm{i}}\right)$ of the whole manufacturing volume. A flowchart of the optimisation procedure is presented in Fig. 5. The optimisation procedure produces a set of greyscale mask images which will be used in MIP-SL manufacturing process.

$F_{(d), P_{i}}=\left\|[T][d]_{P_{i}}-\left[\varphi_{o b j}\right]_{P_{i}}\right\|^{2}$

$[d]^{s+1}{ }_{P_{i}}=[d]_{P_{i}}^{s}-\gamma \cdot \nabla F_{\left(d^{s}\right), P_{i}}$

$\nabla F_{\left(d^{s}\right), P_{i}}=2([T])^{T}\left([T][d]_{P_{i}}^{s}-\left[\varphi_{o b j}\right]_{P_{i}}\right)$

$\left[\varphi_{o b j}\right]_{P_{i}}$ is the objective photopolymerisation variable value to be achieved for pixel domain $P_{i}, s$ is the iterative step number and $\gamma$ is the iterative step size.

The solution obtained from the optimisation algorithm has to be feasible in terms of manufacturing. Therefore, several constraints have to be applied in the iterative algorithm:

(i) All nodal doses have to be positive: $d_{k, P_{i}} \geq 0 \quad \forall k$

(ii) The maximum nodal dose is limited in order to reduce pixel blending: $d_{k, P_{i}} \leq d_{\max } \forall k$

(iii) During the manufacturing, each layer has to be bonded to the previously built-up layer; otherwise the manufacturing process would fail. Therefore, all non-null nodal doses have to fulfil the following requirement (Eq. 10): 


$$
d_{\text {min }} \geq \frac{\exp \left(\mu L_{e} n_{z}\right) \cdot \ln \left(\frac{1}{1-\Phi_{c}}\right)}{K} \quad \forall d_{k, P_{i}} \neq 0
$$

Where $n_{z}$ is the number of elements between non-null nodal applied doses.

A $Z$ axis discretization is done for each pixel domain (Fig. $4(b))$ with a length element $\left(L_{e}\right)$ equal to thickness layer. Once the mesh is obtained, $\left[\varphi_{o b j}\right]_{P_{i}}$ column vector can be defined. Consequently, the optimisation algorithm allows to:

(i) Reduce dimensional errors along manufacturing direction due to non-desired converted layers in any down-facing surfaces.

(ii) Control the photopolymerisation variable of each spatial location, enabling the chance to obtain a more uniform conversion gradient along all 3D printed part homogenizing their mechanical properties and shrinkage along the manufacturing direction.

There is a minimum achievable value for the photoconversion ratio according to the layer thickness $\left(L_{e}\right)$, the threshold conversion ratio $\left(\Phi_{c}\right)$ and the material attenuation factor $(\mu)$ due to the minimum energy dose $\left(d_{\min }\right)$ required to bond each layer. This minimum photoconversion value is a consequence of the total accumulated dose. This value is around 0.939 for a layer thickness of 50 microns. Therefore, a photoconversion ratio of 0.95 has been defined. The theoretical range of photopolymerisation variable value can be determined by Eq. (11). However, it is not recommended to define a full material photoconversion because $\varphi_{o b j}$ would tend to $-\infty$.

$\Phi \in[0,1] \rightarrow\left\{\begin{array}{c}\varphi=\ln (1-0)=0 \\ \varphi=\ln (1-1)=-\infty\end{array}\right\} \rightarrow \varphi \in[-\infty, 0]$

On the other hand, a null $\varphi_{o b j}$ should be defined for all non-converted nodes. Nevertheless, a penalty coefficient $(a)$ could be used to achieve a higher $Z$ resolution (Eq. 12). The penalty coefficient must be positive; thus the objective photopolymerisation variable could never be reached. As a result, the obtained solution could present a higher geometrical accuracy to the detriment of photoconversion ratio uniformity along manufacturing direction. The influence of this coefficient is discussed in Section 4.2 .

$\varphi_{o b j}=a \rightarrow \forall k \in \Phi_{k}=0$ 


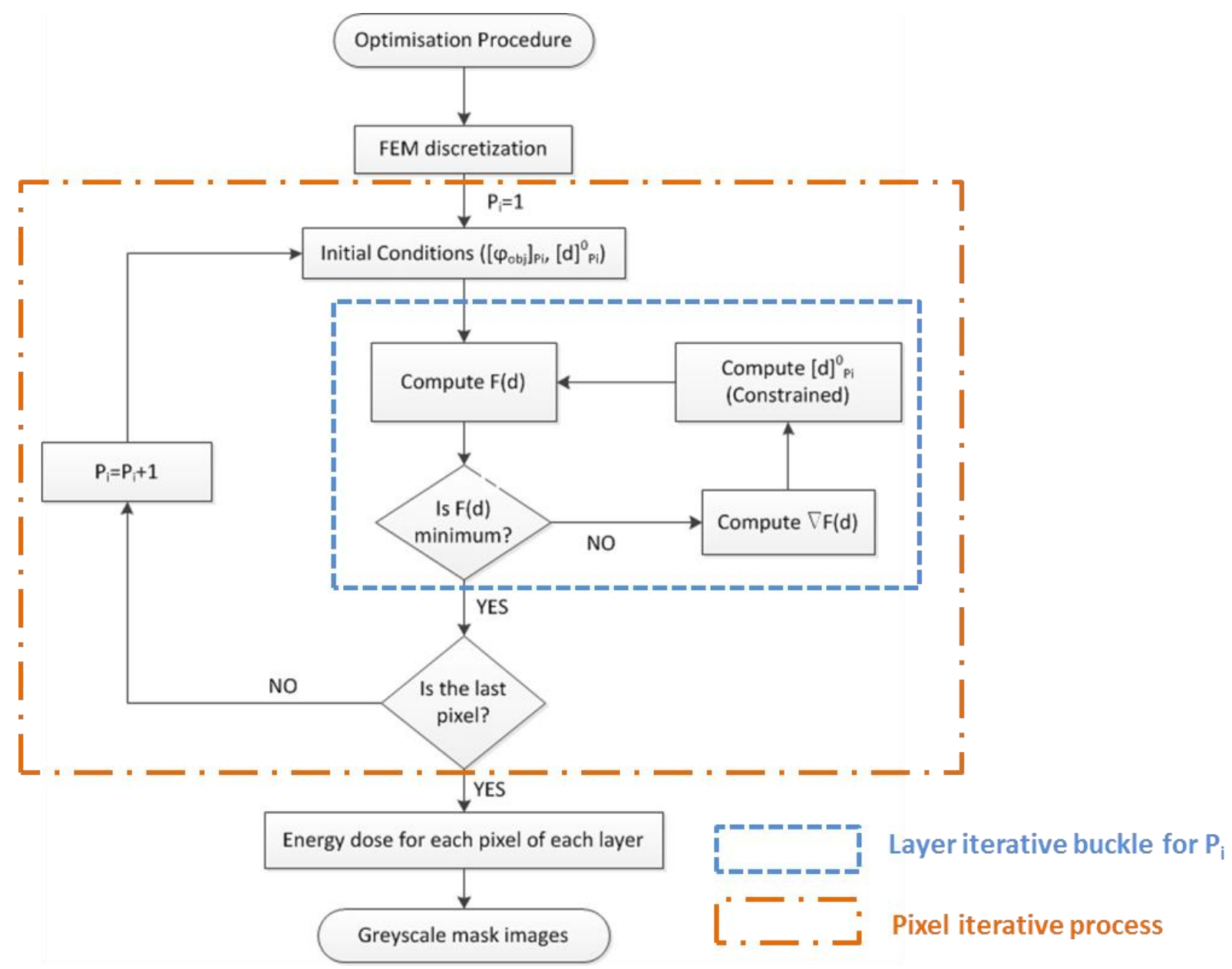

Figure 5 - Flowchart of the optimisation procedure. The blue box corresponds to the layer iterative buckle done along the manufacturing direction for each pixel whereas the orange box corresponds to the iterative buckle done for the whole manufacturing domain.

\subsection{Optimised mask image projection}

Different greyscale levels for each mask image are used in order to define the appropriate exposure dose of each pixel for each layer and, consequently, to optimise the total accumulated exposure dose. The light intensity in function of pixel greyscale level has been obtained through experimental tests similar to Section 2.2. For pixel values higher than 150 (with 5 second of exposure time) the conversion ratio reaches the threshold value. The more the pixel greyscale level increases the higher the solidified thickness is achieved. Thus, light intensity could be experimentally calibrated using FPP model equations and experimental test. Fig. 6(a) shows the relationship obtained.

As a result, the optimised mask image uses different greyscale level values for each mask image in order to obtain the optimised total accumulated dose (Fig. 6). 


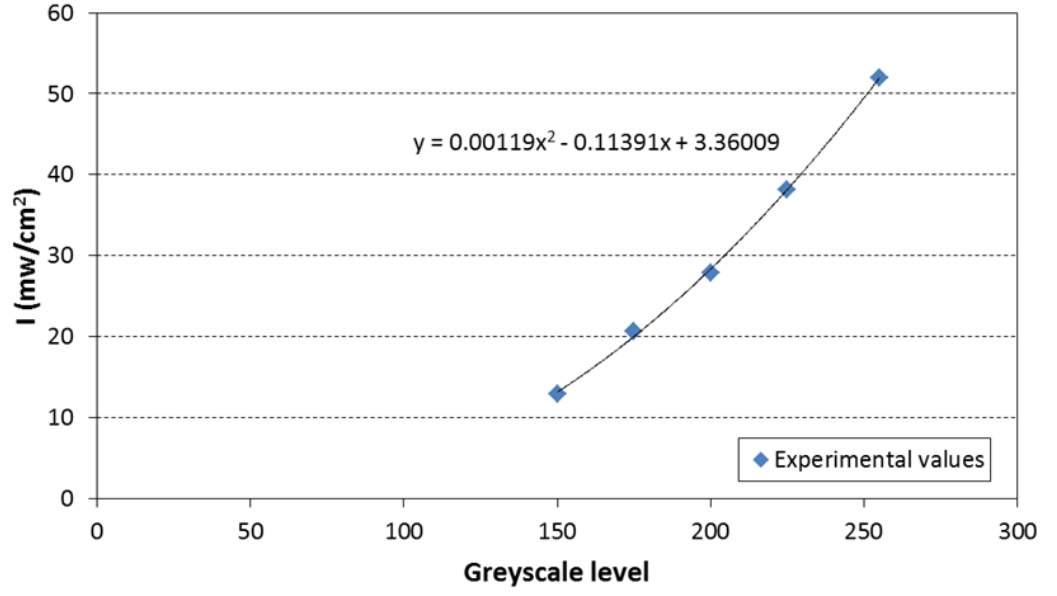

(a)

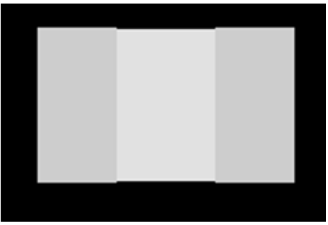

(b)

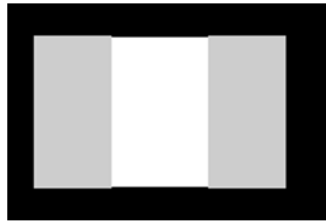

(c)

Figure 6-Experimental relationship between light intensity and pixel greyscale level (a). After the optimisation procedure different pixel greyscale levels are projected in single mask image to enhance $Z$ accuracy of down-facing surfaces. 205-225 greyscale levels are used in (b) and 205-255 in (c)

\section{Discussion of the results}

\subsection{Objective parts}

In order to evaluate the efficiency of the developed optimisation algorithm two different geometries have been specifically designed. Z accuracy and resolution has been obtained through the dimensional measurement of part holes (top and bottom) and part overhangs (top and bottom) for case 1 and 2 respectively. Fig. 7 and 8 show the main $Z$ dimensions (in $\mathrm{mm}$ ) as well as the built-up orientation of objective parts. A layer thickness of 50 microns has been used in the model discretization.
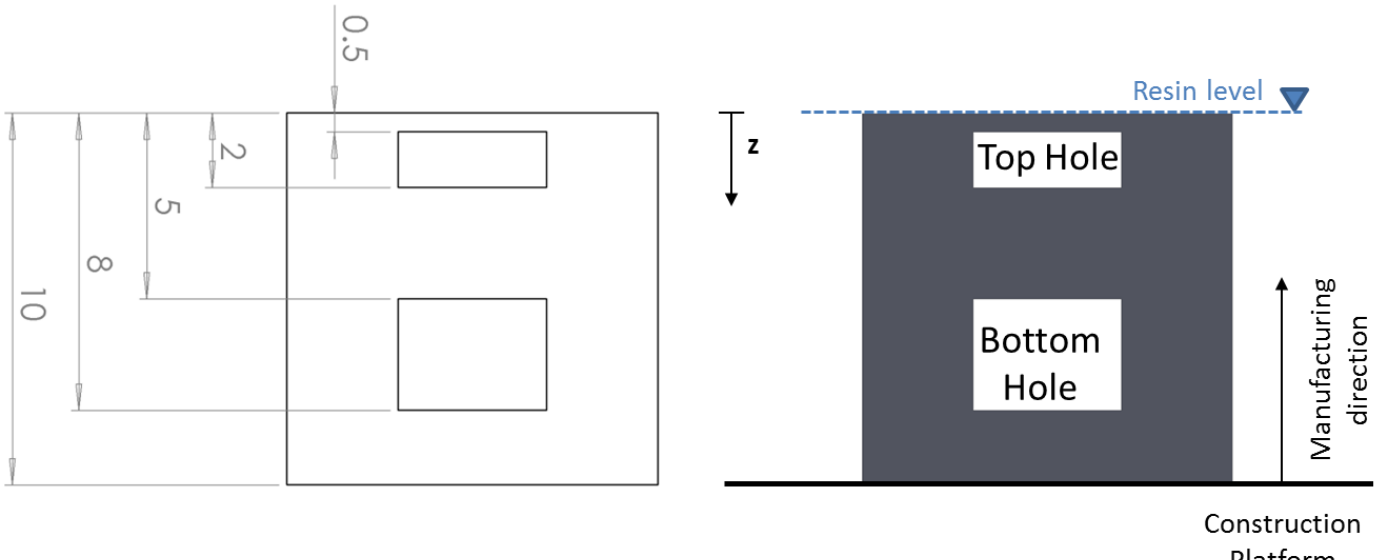

Figure 7-Geometrical dimensions (in $\mathrm{mm}$ ) of case 1 part. The optimisation procedure efficiency would be evaluated through holes height measurements. 


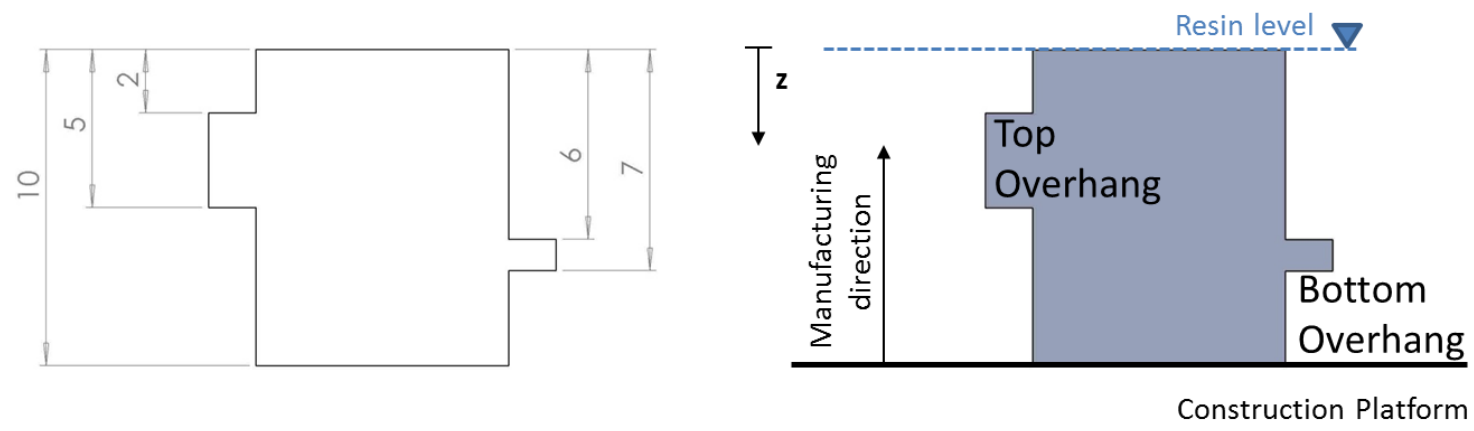

Figure 8 - Geometrical dimensions (in $\mathrm{mm}$ ) of case 2 part. The efficiency of the optimization procedure would be analysed by means of unsupported overhangs height measurements.

\subsection{Influence of penalty coefficient}

First of all, the influence of penalty coefficient in the optimisation algorithm has been analysed. Fig. 9 shows the $\mathrm{Z}$ geometrical difference between the optimised and objective holes/overhangs dimensions for case 1 and case 2, respectively. It can be observed that higher geometrical differences are obtained for low penalty coefficients. In addition, the optimised results tend to be very similar and close to the objective values when the penalty coefficient is higher than 5 . The maximum difference in geometrical dimensions for penalty coefficients higher than 5 is less than 150 microns. Consequently, the use of a penalty coefficient higher than 5 is recommended in order to obtain printed parts with a higher $\mathbf{Z}$ accuracy. For the following sections, a non-null penalty coefficient of 5.5 has been used in the optimisation procedure.

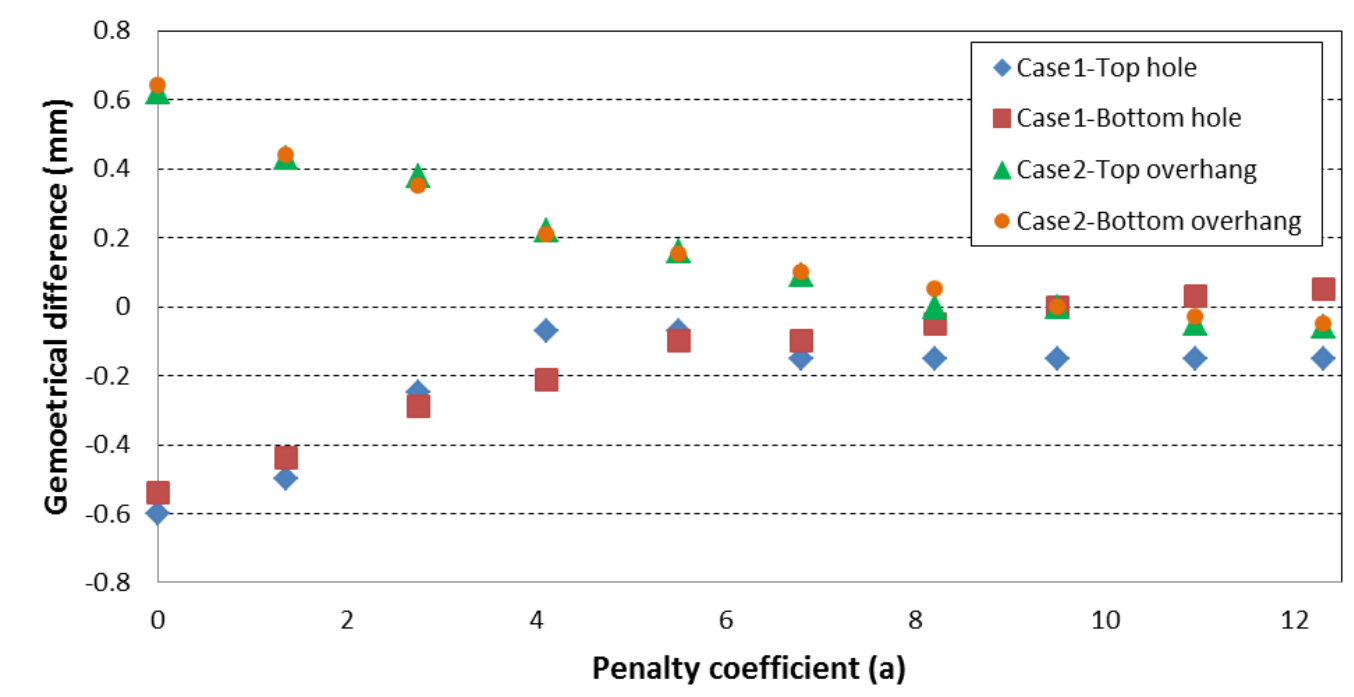

Figure 9-Influence of optimisation penalty coefficient in Z accuracy

The photoconversion ratio distributions of case 1 objective part for a non-null $(a=5.5)$ and a null penalty coefficient are compared in Fig. 10. The conversion distributions have been obtained for a cross-section that takes into account both holes. As it can be seen in Fig. 10, a photoconversion ratio closer than the objective distribution for converted layers could be obtained if a null penalty coefficient is used in the iterative algorithm. However, a lower geometrical accuracy in Z dimensions is achieved. 


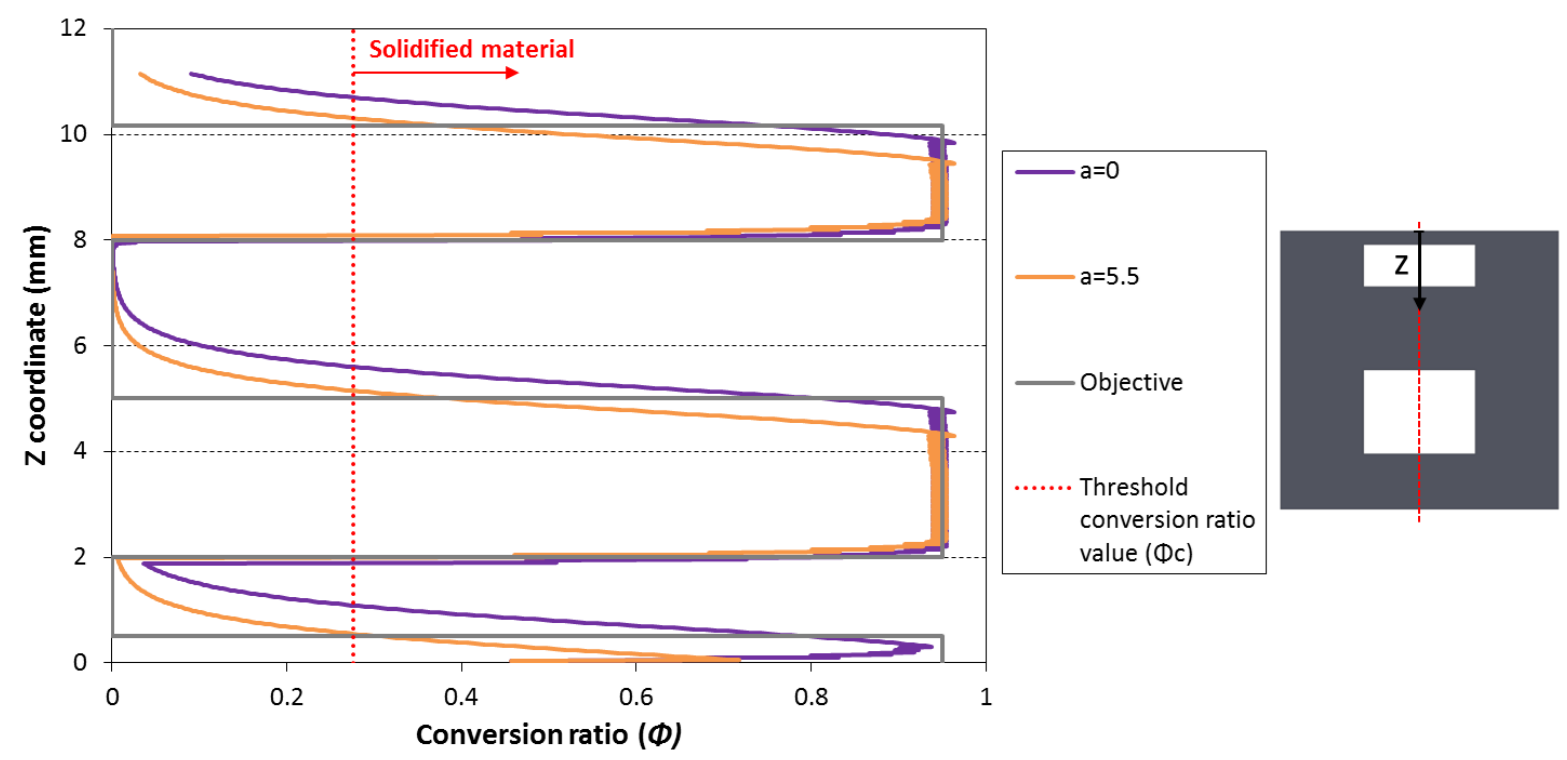

Figure 10 - Objective and numerical photoconversion distribution obtained with different penalty coefficients for case 1 part.

\subsection{Influence of photoconversion ratio range}

Two different optimisations have been done using a non-null penalty coefficient. A maximum exposure dose of $260 \mathrm{~mJ} / \mathrm{cm}^{2}$ and a wide range of conversion ratios along manufacturing direction have been defined for the first optimisation (Opt 1). On the other hand, a maximum dose of $130 \mathrm{~mJ} / \mathrm{cm}^{2}$ and a more uniform conversion ratio has been imposed for the second procedure (Opt 2). Both optimised solutions show a similar behaviour in terms of manufacturing accuracy as it can be observed in Fig. 11. However, Fig. 12 shows that Opt 1 solution presents a reduction of $X Y$ accuracy due to pixel blending effect. Consequently, Opt 2 parameters have been defined to obtain the optimised solution in following sections. The pixel blending effect among layers is not significant for this maximum dose and uniform photoconversion ratio gradient.

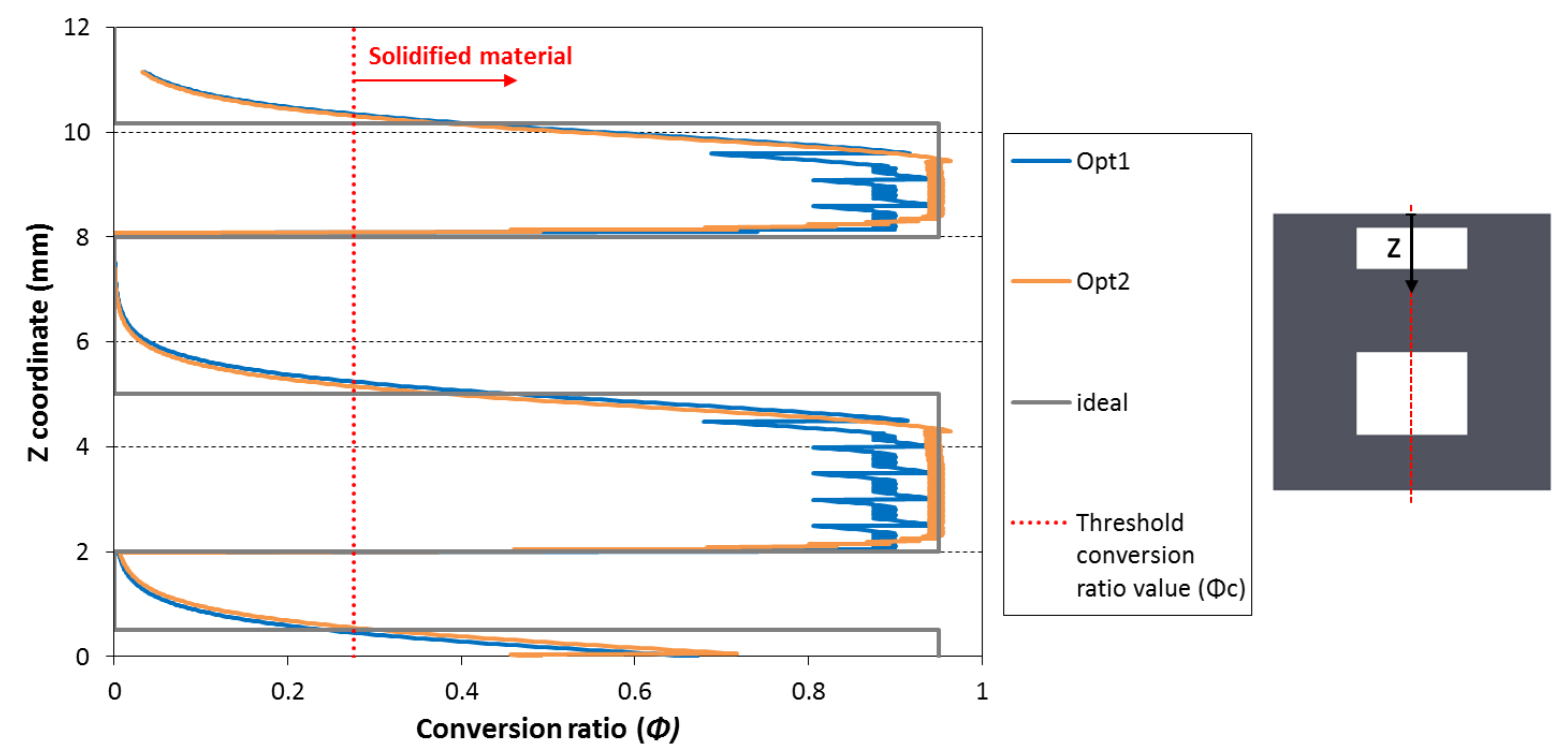

Figure 11 - Objective and numerical photoconversion distribution obtained with different maximum exposure dose and conversion gradient range for case 1 part. 


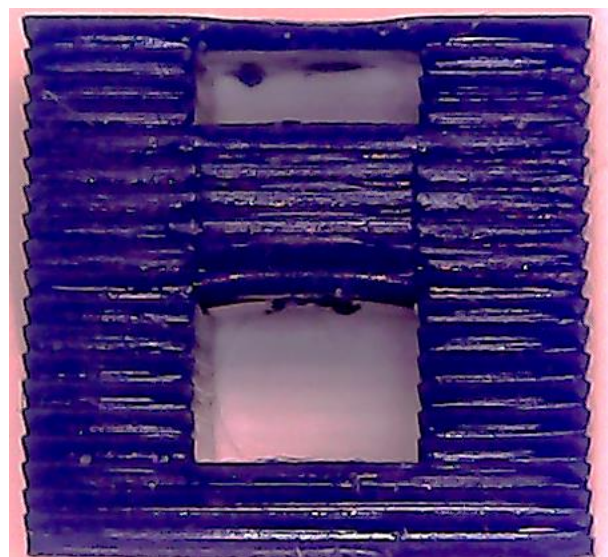

Figure 12 - Stepped solidified pattern obtained as a consequence of pixel blending effect in Opt1 for case 1 part

\subsection{Numerical solution of optimised and non-optimised models}

For the non-optimised models an exposure dose of $78 \mathrm{~mJ} / \mathrm{cm}^{2}$ has been used for all pixels of each layer to obtain a photoconversion ratio mean of solidified parts similar to the objective input (0.95). For case 1 part, the comparison of numerical conversion ratio and exposure dose along $Z$ direction for optimised and non-optimised models is shown in Fig. 13 and 14. The non-optimised solution tends to produce a lower $Z$ accuracy than the optimised one due to the uncontrolled conversion of several layers. The pixel exposure dose for optimised solution is not constant among layers unlike the non-optimised one; consequently different greyscale levels are used for each mask image in each layer. On the other hand, Fig. 15 and 16 show the numerical conversion ratio and exposure dose along the manufacturing direction for the top overhang cross-section for case 2 part.

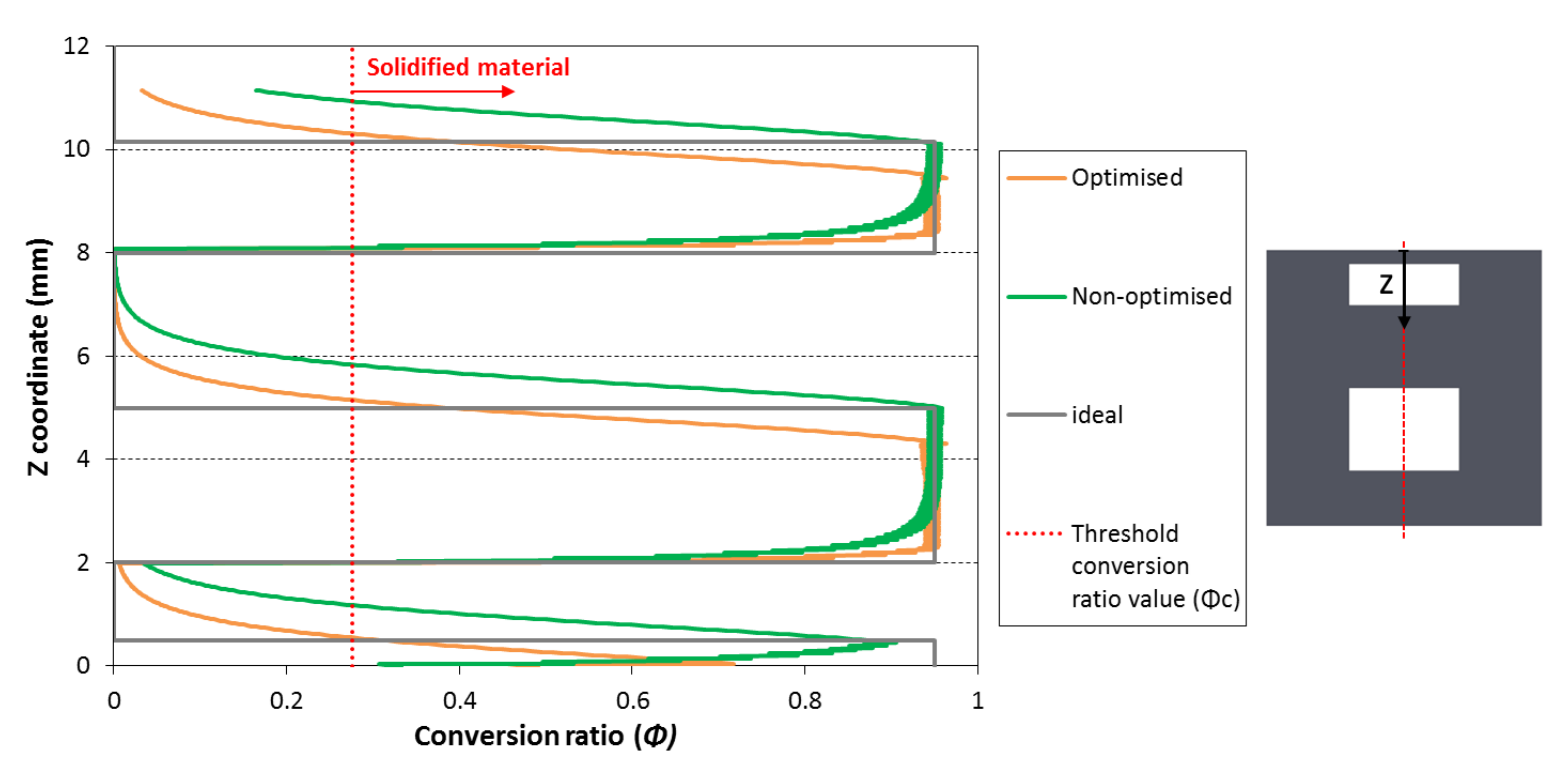

Figure 13 - Objective and numerical photoconversion distributions obtained with optimised and non-optimised solution for case 1 part. 


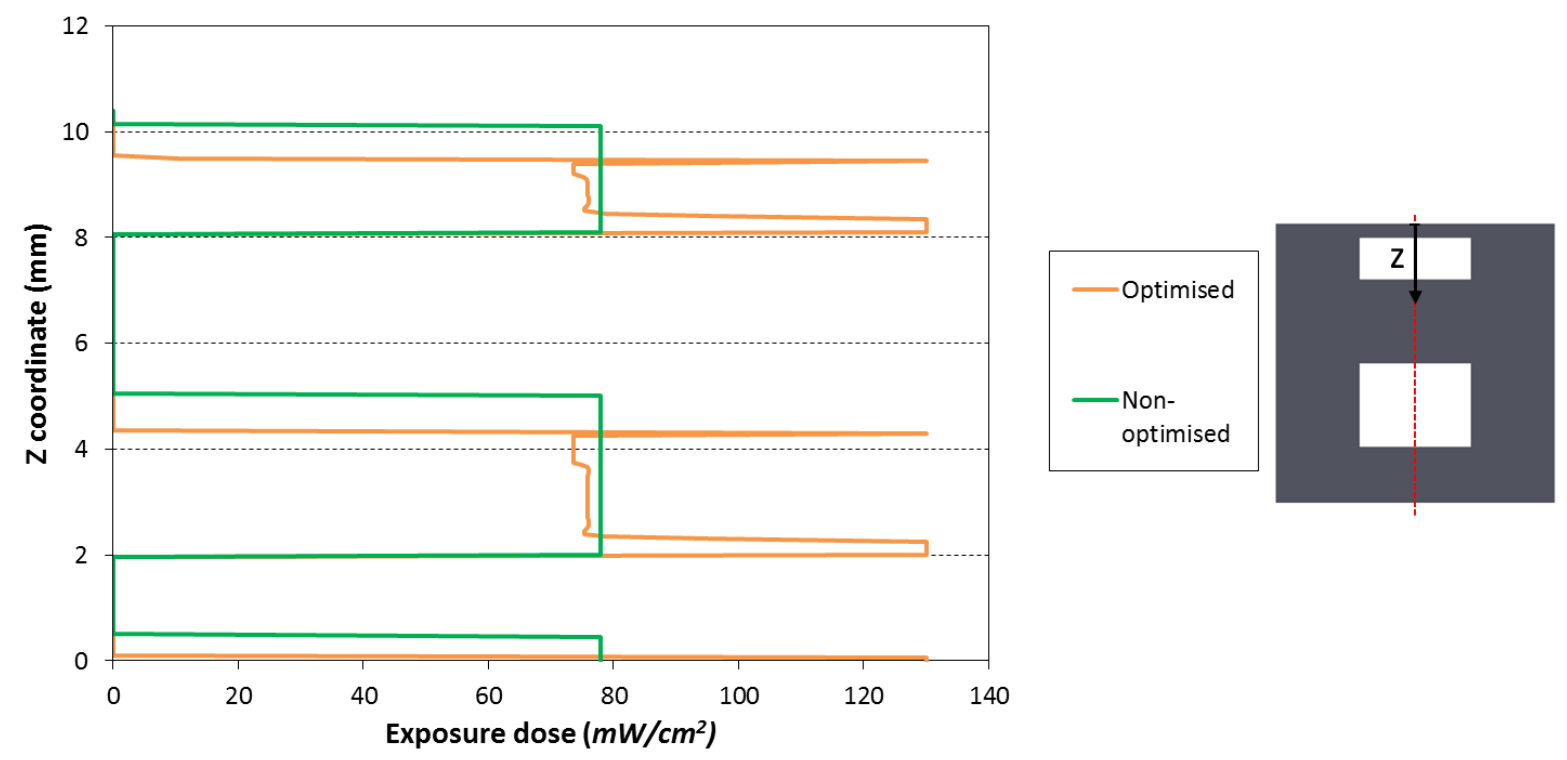

Figure 14 -Optimised and non-optimised exposure dose distribution for case 1 part. Different pixel greyscale level are used in the optimised solution to modify the exposure dose of each layer.

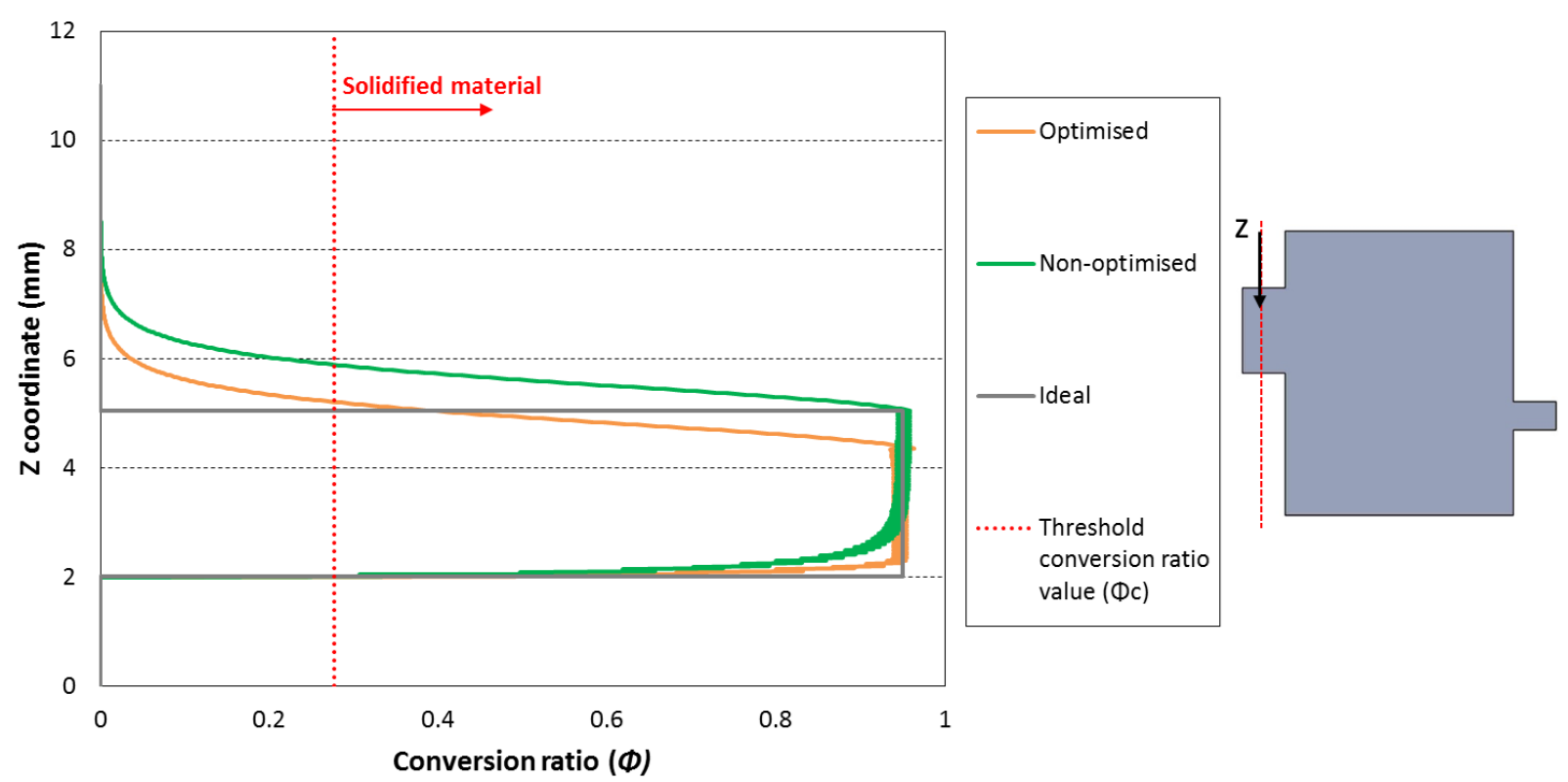

Figure 15 - Objective and numerical photoconversion distributions obtained with optimised and non-optimised solution for case 2 part. 


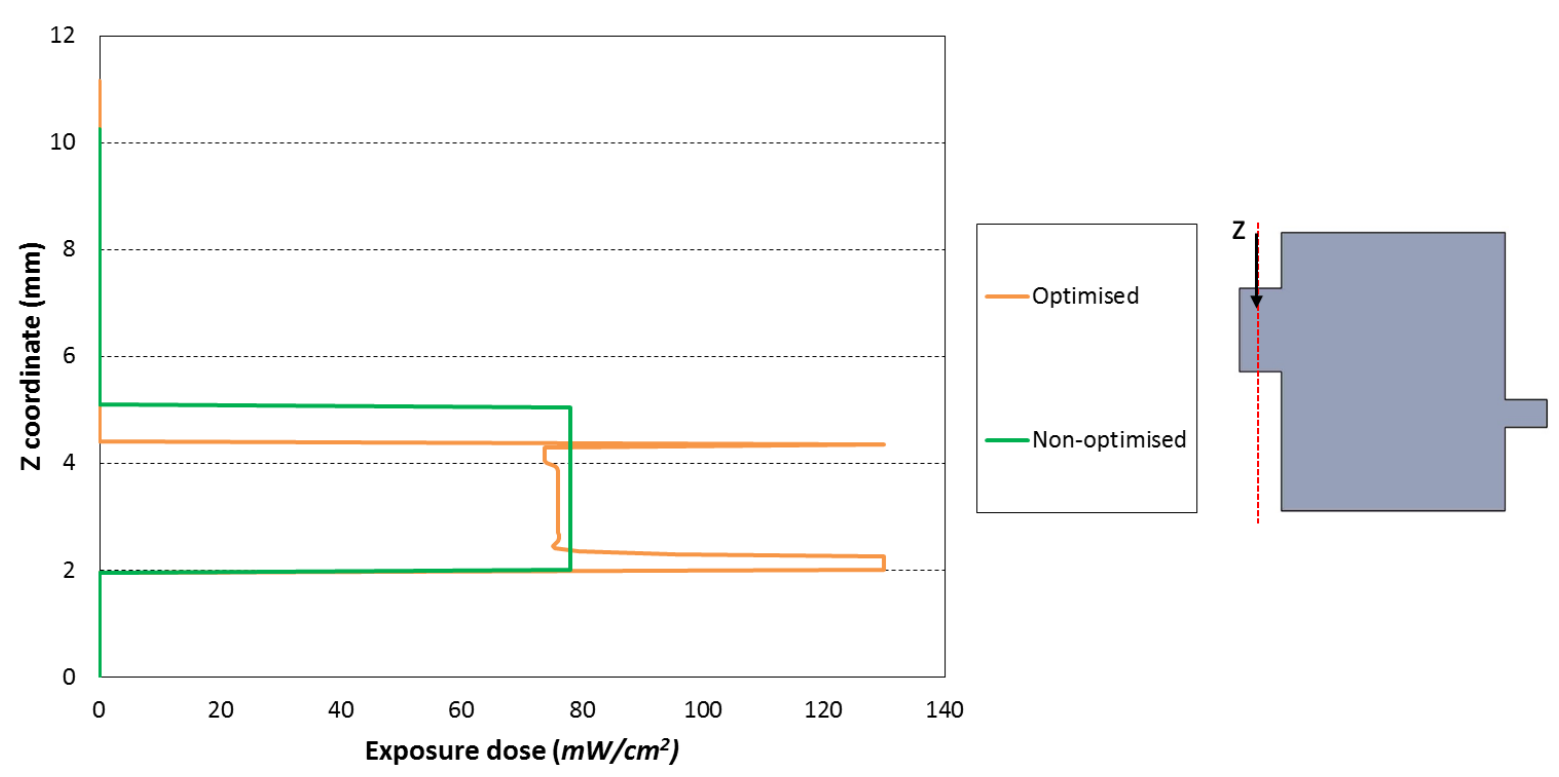

Figure 16 - Optimised and non-optimised exposure dose distribution for case 2 part. Different pixel greyscale levels are used in the optimised solution to modify the exposure dose of each layer.

\section{Experimental results}

\subsection{MIP-SL equipment and printed samples}

Two different configurations are commonly used as MIP-SL equipment: Bottom-Up (Fig. 17(a)) and TopDown (Fig. 17(b)). The main difference between both configurations is the position of the light emission source and the direction of the manufacturing axis. A Top-Down configuration has been used in this paper to avoid the detachment of the cured layer from the resin vat surface through a tilting separation process [27]. A visible light source with a pixel resolution of 62.5 microns has been used.
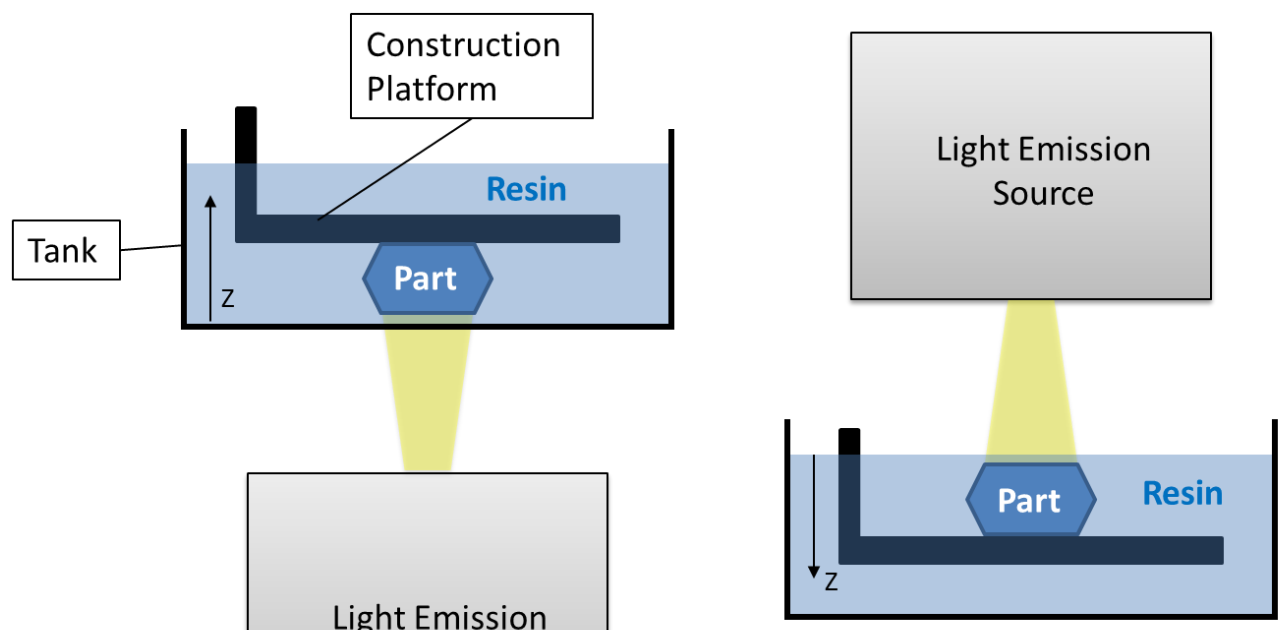

Light Emission

Source

(a)

(b)

Figure 17 - Bottom-up (a) and Top-down MIP-SL (b) configuration 
In order to analyse the influence of the optimisation procedure all samples have been printed with the same parameters: a layer thickness of 50 microns, a controlled temperature of 23 o $C$ and the same location in the built chamber (centre of construction platform). The material used is a commercial photocurable resin (SPOT-HT) based on acrylate oligomers with an absorption spectrum from 385 to 425 $\mathrm{nm}$.

Several experimental tests have been done to validate the optimisation algorithm. Three different samples have been printed with and without the optimisation procedure for each geometry case. Fig. 18 and 19 show the printed and the objective parts for case 1 and 2, respectively. It can be observed a significant difference in holes and overhangs measurements, whereas the others dimensions are practically the same. Consequently, the use of the optimisation procedure only affects Z accuracy as long as the maximum exposure dose is not much higher than the minimum required. Otherwise, a lower $X Y$ resolution could be obtained because of pixel blending (Section 4.3).

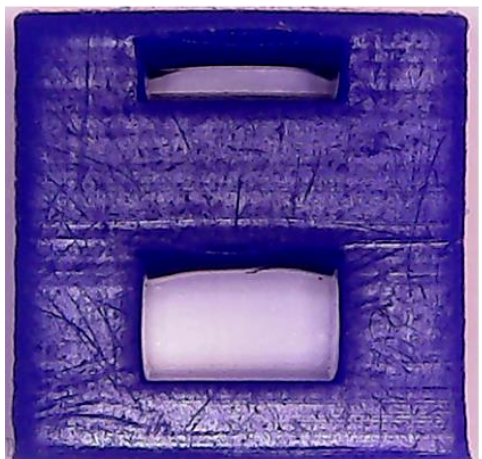

(a)

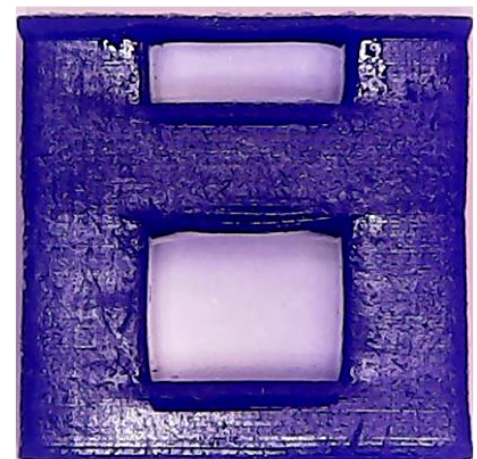

(b)

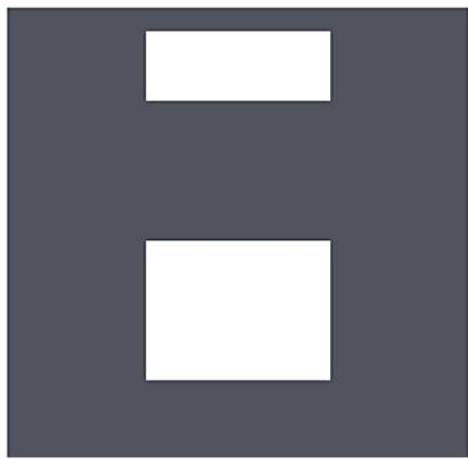

(c)

Figure 18 - Case 1 parts obtained without (a) and with the optimisation procedure (b). Objective part (c). A significant difference in $Z$ dimension is measured.
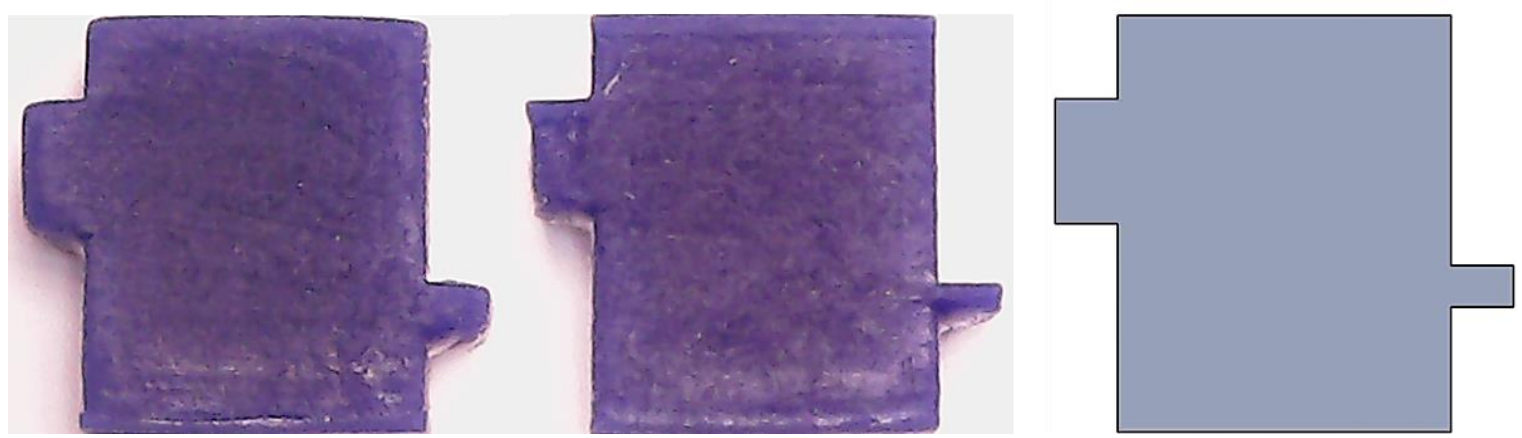

Figure 19 - Case 2 parts obtained without (a) and with the optimisation procedure (b). Objective part (c). A higher dimensional accuracy is obtained in optimised parts.

\subsection{Comparison of objective values, numerical estimations and printed samples}

Table 2 shows the objective (in brackets) and the experimental dimensions for all printed samples. In Fig. 20 the $Z$ dimensional errors among experimental measurement and objective values are plotted. The $Z$ dimensional accuracy is increased as a result of the application of the optimisation algorithm. The mean absolute error for non-optimised parts is around $0.66 \mathrm{~mm}$ whereas for the optimised ones is 0.08 $\mathrm{mm}$. There is no significant difference in standard deviation between non-optimised $(0.07 \mathrm{~mm})$ and optimised parts $(0.06 \mathrm{~mm})$. Consequently, the results show an improvement of $Z$ accuracy for optimised parts.

In addition, the increase of non-desired solidified layers in non-optimised printed parts could be different in all down-facing surfaces because it depends on the total accumulated dose. Thus, 
dimensional errors in bottom holes are higher than the top ones for case 1 parts. Consequently, the same dimensional modification of all down-facing surfaces in STL file could not produce a geometrical solution as optimal as through the developed optimisation procedure.

\begin{tabular}{|c|c|c|c|}
\hline \multirow[b]{2}{*}{ Sample } & \multirow[b]{2}{*}{ Type } & \multicolumn{2}{|c|}{ Experimental and objective (in brackets) values ( $\mathrm{mm}$ ) } \\
\hline & & Top measurement & Bottom measurement \\
\hline S1 & Case 1 - non-optimised & $0.85(1.5)$ & $2.2(3)$ \\
\hline S2 & Case 1 - non-optimised & $0.92(1.5)$ & $2.29(3)$ \\
\hline s3 & Case 1 - non-optimised & $0.94(1.5)$ & $2.32(3)$ \\
\hline S4 & Case 2 - non-optimised & $3.7(3)$ & $1.72(1)$ \\
\hline S5 & Case 2 - non-optimised & $3.59(3)$ & $1.67(1)$ \\
\hline S6 & Case 2 - non-optimised & $3.65(3)$ & $1.64(1)$ \\
\hline S7 & Case 1 - optimised & $1.35(1.5)$ & $2.85(3)$ \\
\hline S8 & Case 1 - optimised & $1.31(1.5)$ & $3.02(3)$ \\
\hline S9 & Case 1 - optimised & $1.39(1.5)$ & $2.96(3)$ \\
\hline S10 & Case 2 - optimised & $3.01(3)$ & $1.05(1)$ \\
\hline S11 & Case 2 - optimised & $2.98(3)$ & $1.07(1)$ \\
\hline $\mathrm{S} 12$ & Case 2 - optimised & $2.95(3)$ & $1.11(1)$ \\
\hline
\end{tabular}

Table 2 - Experimental Z dimensions for all printed samples. Objective values are in brackets.

In order to evaluate the accuracy of the analytical model, the difference between printed parts dimensions and numerical estimations are presented in Fig. 21. The results provide a good agreement among experimental and numerical values for optimised and non-optimised parts. The mean of the geometrical difference obtained is $-0.06 \mathrm{~mm}$ with a standard deviation of $0.11 \mathrm{~mm}$. Therefore, the optimisation procedure does not decrease the efficiency of the analytical model.

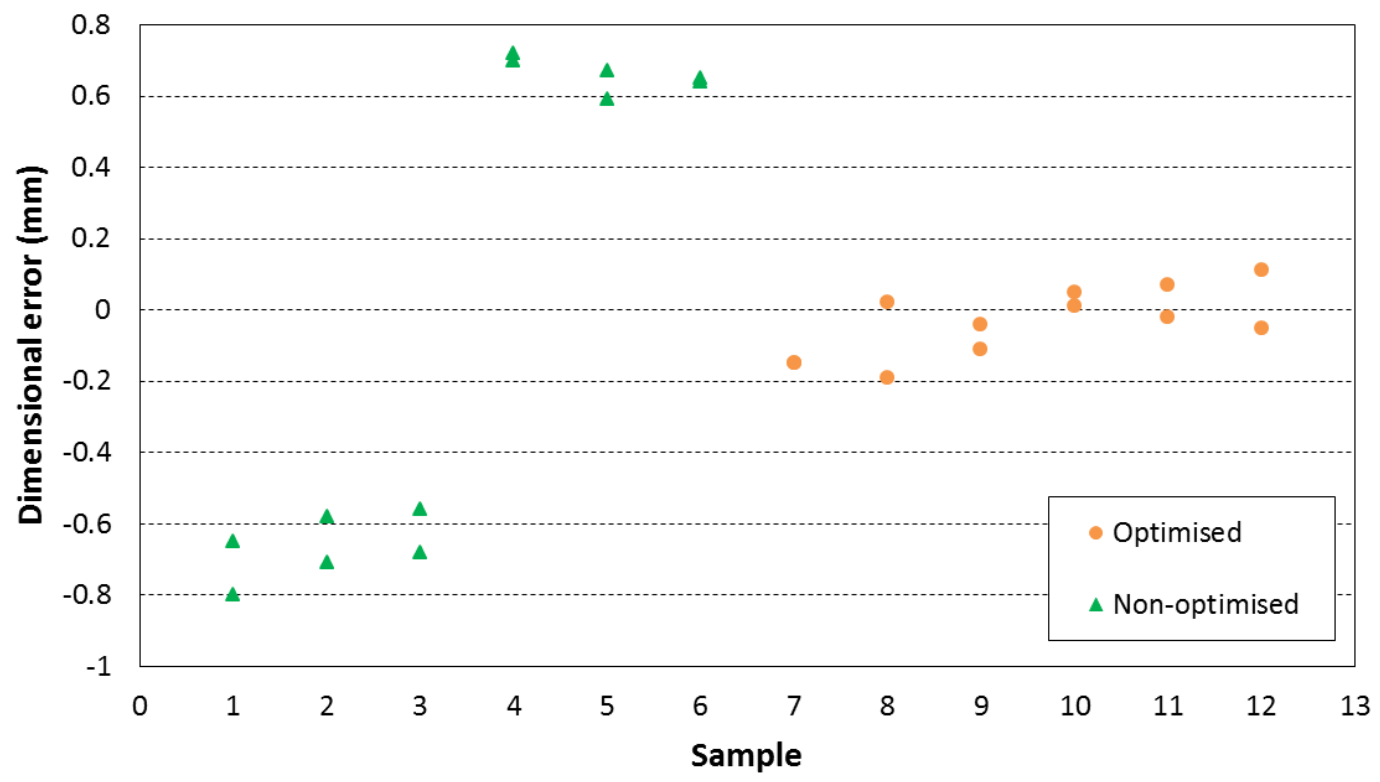

Figure 20-Z dimensional error between experimental and objective measurements 


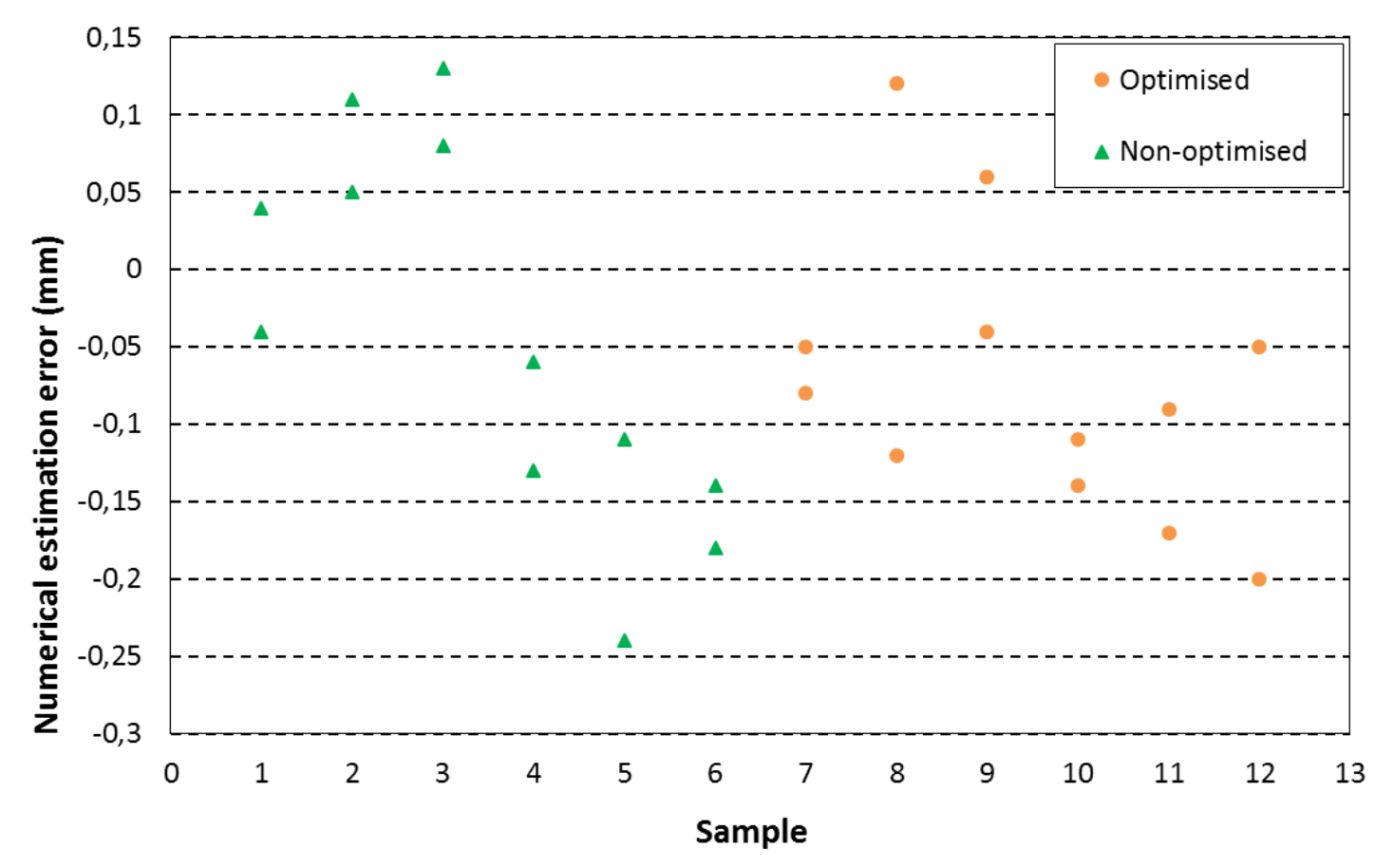

Figure $21-Z$ dimensional error between numerical estimations and printed parts

\section{Conclusions}

One of the main problems in mask image projection based on stereolithography (MIP-SL) is the noncontrolled light energy penetration into the photocuring material. As a result, $Z$ accuracy of printed parts could be affected. In this paper a novel optimisation procedure based on a finite element method to control the photoconversion ratio and to increase manufacturing accuracy of printed parts is presented.

First of all, a 2-node finite element has been implemented based on FPP model to discretize the manufacturing volume in several subdomains. Consequently, the photoconversion ratio for all subdomains could be calculated in function of each nodal exposure dose. Furthermore, this finite element could be used for the optimisation procedure.

The optimisation procedure implemented increases $Z$ accuracy due to the reduction of non-desired converted layers considering the total accumulated doses received in each spatial location. The model takes into account the main photocuring material parameters; thus it can be easily adapted to different photosensitive materials. Moreover, the non-controlled light energy penetration could be different in function of the down-facing upper layers. Consequently, this solution could provide more effective results than a simple modification of down-facing surfaces in STL file.

In addition, the presented methodology allows to control the photoconversion ratio of any spatial location of printed parts; therefore it is possible to define an objective conversion ratio along the manufacturing direction obtaining a more uniform conversion profile or near to a full conversion.

Two different parameters have a relevant role in the optimisation algorithm. The penalty coefficient allows a prioritization between $Z$ accuracy or a conversion ratio closer to the objective ratio value. On the other hand, the maximum exposure allowed as well as the conversion gradient could have a significant influence on $\mathrm{XY}$ resolution. A high exposure dose produces an increase of in-plane solidified 
areas due to pixel blending effect. Therefore, a large variation between maximum and minimum exposure dose or conversion ratios is not advisable if the pixel blending is not considered.

To sum up, a novel optimisation procedure based on the control of the photocuring conversion ratio to increase $Z$ accuracy is successfully developed and implemented. The algorithm provides a specific exposure dose for each pixel of each layer via different greyscale mask images. The experimental results show a good agreement with numerical estimations.

\section{Acknowledgments}

Authors (1) and (2) would like to thank the Spanish Ministry of Economy and Competitiveness for its financial support for the research project "New technologies for 3D printing of advanced materials (DPI2016-80119-C3-1-R)" and "New multifunctional thermosets obtained by dual curing (MAT201453706-C03-02)", respectively (AEI/FEDER, UE).

\section{References}

[1] Bikas H, Stavropoulos P, Chryssolouris G. Additive manufacturing methods and modelling approaches: a critical review. The international Journal of Advanced Manufacturing Technology (2016), 83: 389-405.

[2] Singh S, Ramakrishna S, Singh R. Materials issues in additive manufacturing: A review. Journal of Manufacturing Processes (2017), 25: 185-200.

[3] Stroud I, Xirouchakis P.C. STL and extensions. Advances in Engineering Software (2000), 31: 83-95.

[4] Ivanov V, Decker C. Kinetic study of photoinitiated frontal polymerization. Polymer International (2001), 50: 113-118.

[5] Hayki N, Lecamo L, Désilles N, Lebaudy P. Kinetic study of photoinitiated frontal polymerization. Influence of UV light intensity variations on the conversion profiles. Macromolecules (2010), 43: 177184.

[6] Cabral J, Douglas J. Propagating waves of network formation induced by light. Polymer (2005), 30, 4230-4241.

[7] Vitale A, Cabral J. Frontal Conversion and Uniformity in 3D Printing by Photopolymerisation. Materials (2016), 9, 760.

[8] Vitale A, Henessy M.G, Matar O.K, Cabal J. Interfacial profile and propagation of frontal photopolymerization waves. Macromolecules (2015), 48, 198-205.

[9] Sun C, Fang N, Wu D.M, Zhang X. Projection micro-stereolithography using digital micro-mirror dynamic mask. Sensors and Actuators A: Physical (2005), 121: 113-120.

[10] Limaye A.S, Rosen D.W. Process planning for mask projection stereolithography. Rapid Prototyping Journal (2007), 13: 76-84.

[11] Zhou C, Chen Y. Additive manufacturing based on optimized mask video projection for improved accuracy and resolution. Journal of manufacturing processes (2012); 14: 107-118. 
[12] Zhou C, Chen Y, Waltz R.A. Optimized mask image projection for solid freeform fabrication. ASME journal of manufacturing science and engineering (2009); 131, 061004-1-12.

[13] West A.P, Sambu S.P, Rosen D.W. A process planning method for improving build performance in stereolithography. Computer-Aided Design (2001); 33:65-79.

[14] Zha W, Anand S. Geometric approaches to input file modification for part quality improvement in additive manufacturing. Journal of Manufacturing processes (2015); 20:465-477.

[15] Manmadhachary A, Ravi Kumar Y, Krishnanand L. Improve the accuracy, surface smoothing and material adaption in STL file for RP medical models. Journal of Manufacturing Processes (2016); 21: 4655.

[16] Pan Y, Zhao X, Chen Y. Smooth surface fabrication in mask projection based stereolithography. Journal of manufacturing processes (2012); 14: 460-470.

[17] Lee J.H, Prud'homme R.K, Aksay I.A. Cure depth in photopolymerization: Experiments and theory. J. Mater. Res., Vol. 16, No. 12, Dec 2001.

[18] Salmoria G.V, Ahrens C.H, Beal V.E, Pires A.T.N, Soldi V. Evaluation of post-curing and laser manufacturing parameters on the properties of SOMOS 7110 photosensitive resin used in stereolithography. Materials and design (2009), 30, 758-763.

[19] Choi J.W, Wicker R.B, Cho S.H, Ha C.S, Lee S.H. Cure depth control for complex 3D microstructure fabrication in dynamic mask projection microstereolithography. Rapid Prototyping Journal (2009); 15: 59-70.

[20] ALimaye A.S, Rosen D.W. Compensation zone approach to avoid print-through errors in mask image projection stereolithography builds. Rapid Prototyping Journal (2006), 12(5): 283-291.

[21] Sager B, Rosen D.W. Use of parameter estimation for stereolithography surface finish improvement. Rapid Prototyping Journal (2008), 14(4): 213-220.

[22] Vitale A, Hennessy M.G, Matar O.K, Cabral J. A Unified Approach for patterning via frontal photopolymerization. Advanced Materials (2015), 27, 6118-6124.

[23] Alvankarian J, Majlis B.Y. Exploiting the Oxygen Inhibitory Effect on UV Curing in Microfabrication: A Modified Lithography Technique. PLOS ONE (2015), doi:10.1371/journal.pone.0119658

[24] Fernández-Francos X, Ramis X. Structural analysis of the curing of epoxy thermosets crosslinked with hyperbranched poly(ethyleneimine)s. European Polymer Journal (2015), 70: 286-305.

[25] Dušek K, Šomvársky J. Modelling of ring-free crosslinking chain (co)polymerization. Polymer International (1997), 44(3): 225-236.

[26] Pascault, J.P, Sautereau H, Verdu J, Williams R.J.J. Thermosetting Polymers. Marcel Dekker, New York (2002).

[27] Wu X, Lian Q, Li D, Jin Z. Tilting separation analysis of bottom-up mask projection stereolithography based on cohesive zone model. Journal of Materials Processing Technology (2017), 247: 184-196. 